\title{
M-learning en el CELEX-ESIA Z. Estrategia educativa que incorpora el WhatsApp al E-learning para el desarrollo de la competencia oral y escrita del idioma inglés
}

\section{M-learning in CELEX-ESIA Z. Educational strategy that incorporates WhatsApp to E-learning for the development of oral and written English language skills}

\author{
Mercedes Leticia Sánchez Ambriz ${ }^{1}$ \\ Sonia Martínez Balboa ${ }^{2}$ \\ Instituto Politécnico Nacional, México
}

Recibido: 08-03-2018

Aceptado: 11-06-2018

\section{Cita recomendada}

Sánchez, M. \& Martínez, S, (2018). M- Learning en el CELEX-ESIA Z- Estrategia educativa que incorporá el WhatsApp al E-learning para el desarrollo de la competencia oral y escrita del idioma inglés. Hamut'ay, 5 (1), 66-83. http://dx.doi.org/10.21503/hamu.v5i1.1521

\section{RESUMEN}

El presente trabajo tuvo como objetivo evaluar la aplicación del M-learning a través de la incorporación del WhatsApp al E-learning del CELEX-ESIA Z, como una estrategia educativa para el desarrollo de la competencia oral y escrita del idioma inglés. Esta investigación surge al comparar los resultados de la modalidad virtual y presencial que imparte el Centro de Lenguas Extranjeras, de la Escuela Superior de Ingeniería y Arquitectura, Unidad Zacatenco del Instituto Politécnico Nacional, México. De acuerdo con los estudios cuantitativos que se llevaron a cabo, con estudiantes de inglés intermedio nivel 5 de la modalidad virtual, se identificó un desfase en el desarrollo de las habilidades en la conversación oral y escrita en contraste con los estudiantes presenciales, por lo que se decidió integrar el M-learning a través del uso de WhatsApp, en un intento de promover sus aprendizajes.

Esta herramienta de mensajería permitió una mayor comunicación e interacción entre estudiantes-estudiantes y docente-estudiantes, al dar seguimiento y retroalimentación de manera inmediata, a las actividades realizadas en la plataforma educativa de Cambridge University Press, lo que permitió optimizar el desarrollo del programa, al identificar las áreas de oportunidad que ofrece actualmente la tecnología.

Palabras Clave: M-learning, educación en línea, WhatsApp y comunicación oral y escrita en inglés.

\section{Abstract}

The objective of this paper was to evaluate the application of M-learning through the incorporation of WhatsApp into the E-learning of CELEX-ESIA Z, as an educational strategy for the development of the oral and written competence in the English language. This research arises when comparing the

1 Asesora académica de CELEX ESIA, Zacatenco, correo merleti70@gmail.com

2 Coordinadora del CELEX ESIA, Zacatendo, correo smartinezb2@gmail.com 
results of the virtual and the face-to-face modality imparted by the Center for Foreign Languages, of the Higher School of Engineering and Architecture, Zacatenco Unit of the National Polytechnic Institute, Mexico. According to the quantitative studies that were carried out, with level 5 intermediate English students of the virtual modality, a gap was identified in the development of skills in oral and written conversation in contrast to the face-to-face students, which was the reason to integrate M-learning through the use of WhatsApp, as an attempt to promote their learning.

This messaging tool allowed greater communication and interaction between student-student and teacher-students, by giving immediate follow-up and feedback to the activities carried out in the Cambridge University Press educational platform, which allowed to optimize the development of the program, by identifying the areas of opportunity currently offered by technology.

Keywords: M-learning, online education, WhatsApp and oral and written communication in English.

\section{INTRODUCCIÓN}

El Centro de Lenguas Extranjeras (CELEX) de la Escuela Superior de Ingeneriería y Arquitectura de Zacatenco, del Instituto Politécnico Nacional, se fundó en mayo de 2012, para ofrecer cursos intensivos de cuatro horas diarias para la modalidad presencial; impartiendo cuatro de los seis niveles de dominio de una lengua extranjera establecidos en el Marco Común Europeo (A1, A2, B1 y B2), con el apoyo de docentes certificados que acompañan a los estudiantes. Sin embargo, no todos en su mayoría egresados y público en general, estaban en condiciones de integrarse en los cursos presenciales, y como alternativa se diseñó e implementó el Programa de Inglés Virtual (PIV), que buscaba, además, cumplir con el requerimiento de titulación establecido.

Utilizando las ventajas que ofrece el aprendizaje en línea y conservando la calidad de los cursos que imparte CELEX ESIA-Z presencialmente, llevó a la Coordinación de esta institución a investigar e implementar estrategias que permitieran a los estudiantes desarrollar las competencias del idioma inglés con el mismo nivel de competencia comunicativa que en la modalidad presencial. Desde esta perspectiva, se consideró apoyarse en la plataforma educativa de Cambridge University Press, creada para promover el aprendizaje autónomo y se asignó, además, un docente que acompañó al estudiante en este proceso. Una vez finalizado el nivel cinco, se evaluaron ambas modalidades, encontrádose una diferencia significativa menor en la competencia oral y escrita de la modalidad virtual, por lo que se decidió integrar la herramienta del WhatsApp, al considerarla como uno de los medios de comunicación más utilizados y de fácil acceso al ofrecer conectividad, integración multimedia, grabación de textos de voz, mensajes de audio y la inmediatez para la comunicación síncrona y asíncrona, siendo esta una opción innovadora en la adquisición del idioma inglés, por lo que se realizó este estudio, teniendo como objetivo: Evaluar la aplicación del M-learning a través de la incorporación del WhatsApp al E-learning del CELEX-ESIA Z, como una estrategia educativa para el desarrollo de la competencia oral y escrita del idioma inglés.

\section{Telefonía móvil en la educación}

Los avances tecnológicos han permitido el desarrollo de los llamados teléfonos inteligentes, convertidos en pequeñas computadoras, además, de tener la ventaja de conectarse a Internet por medio de redes inalámbricas. Estos dispositivos llevados al aula permiten el acceso a contenidos y experiencias educativas de bajo costo y por lo tanto deberían incorporarse a las clases en lugar de ser prohibidos (Baird et al, 2016; Chevrier et al, 2013; Forinash \& Wisman, 2015; Hall, 2013; Kuhn \& Vogt, 2013; MacIsaac, 2015; 
M-learning en el CELEX-ESIA Z. Estrategia educativa que incorpora el WhatsApp al E-learning para el desarrollo de la competencia oral y escrita del idioma inglés

Mau et al, 2016; Monteiro et al, 2016; Müller et al, 2015; Shakur \& Kraft, 2016; Tornaría, Monteiro \& Marti, 2014; Vogt, Kuhn \& Neuschwander, 2014). Según Motorola (2018) en un estudió realizado reveló que para el $56 \%$ de las personas entrevistadas, de un universo de 4.418 participantes, consideran al smartphone como su mejor amigo, para justificar la adicción a este producto tecnológico. Según la International Telecommunication Union (2016), en los países desarrollados, el $95 \%$ de las personas adultas poseen un teléfono móvil, mientras que en los países en desarrollo la penetración es del 90\%. En México se llevó a cabo la "Primera Encuesta Nacional sobre consumo digital y cultura" (2015), en donde se señaló que el $97 \%$ de los encuestados cuentan con un Smartphone y la mayoría se conecta a citados, al señalar que existen múltiples beneficios en el uso del aprendizaje móvil, como son: 1) motivación, 2) portabilidad, 3) retroalimentación inmediata, 4) libertad en el manejo del tiempo personal y para actividades de aprendizaje. De esta manera, el aprendizaje móvil ofrece una opción innovadora en la adquisición del idioma inglés al utilizar recursos de multimedia por medio de la conectividad a Internet. Asimismo, como se describe en la tabla 1 , según Naismith et al. (2005), hay diversos enfoques pedagógicos que sustentan el uso de estos dispositivos, resumidos en: el constructivista, el situacional, el colaborativo y el informal.

Tabla 1 Corrientes pedagógicas y aplicaciones móviles

\begin{tabular}{llll}
\hline CONSTRUCTIVISTA & \multicolumn{1}{c}{ SITUACIONAL } & COLABORATIVO & INFORMAL \\
\hline El alumno construye & Enfatiza el contexto donde & Utiliza los mecanismos para & Las aplicaciones móviles de- \\
su propio conocimiento & estén inmersos, los estudian- & las interacciones entre los ben ofrecer vías para adquirir \\
basado en nuevas ideas & tes y se presenta información & involucrados en el proceso y el conocimiento en un esque- \\
y conocimientos previos, & dependiendo de la situación, & resalta los medios utilizados ma más libre, en donde las \\
por lo que las aplicaciones & lugar o tiempo donde se en- & para comunicarse entre sí, actividades no necesariamen- \\
móviles deberán ofrecer & cuentre el alumno. & y utilizando mecanismos de te dependen de un currículo y \\
esquemas de virtualización & coordinación de tareas o gru- generalmente las experiencias \\
de contextos, y herramien- & pos. En adición a esto, toda la se dan fuera del salón de clase \\
tas que permitan administrar & teoría del cómputo colaborati- \\
dicho conocimiento, así & vo puede ser vertida en este \\
como métodos de búsqueda & modelo, a través de las tecno- \\
de información relevante al & logías móviles, sin implicar un \\
problema planteado. & sustituto a las interacciones \\
& hombre-maquina. \\
\hline
\end{tabular}

Internet con servicios de prepago; en el caso de los universitarios 4 de cada 10 cuentan con un plan de pago. Asimismo, en el estudio realizado por iLifebeht Time, México, en mayo del 2017, respecto al uso de aplicaciones, se evidenció que los mexicanos estaban 35 horas semanales en línea y que los llamados "millenial", dedicaban 37 horas a esta actividad, y los sitios más visitados eran las redes sociales y la mensajería instantánea, colocando al WhatsApp como la aplicación más utilizada.

Dentro de este contexto es ineludible encontrar la mejor forma para aprovechar el WhatsApp como herramienta en el ámbito educativo, sobre todo, para acompañar a los jóvenes en su proceso de apropiación del conocimiento y en especial en el aprendizaje del idioma inglés. Por su parte, Ally \& Samaka (2013) coinciden con los autores antes
Fuente: Naismith et al (2005). Adaptación.

\section{E-Learning}

El E-Learning es una modalidad educativa que surge con el desarrollo de las nuevas Tecnologías de la Información e Internet. Consiste en aprovechar la facilidad de distribución de materiales formativos y las herramientas de comunicación que ofrece la Red para crear un entorno para el aprendizaje.

Mediante esta tecnología el estudiante tiene acceso a cursos interactivos y multimedia en formato Web, apoyados con medios de comunicación que permiten la colaboración y discusión online de las materias estudiadas. Estos mismos medios permiten que la formación sea tutorizada por un experto que realice un seguimiento del progreso 
de los estudiantes, así como la orientación, resolución de dudas, motivación, etc. (Moreno, 2013).

Dentro de las principales ventajas del E-learning debe destacarse la facilidad de acceso. La formación puede llegar a más personas, puesto que desaparecen las barreras espaciotemporales. De esta forma, personas que antes tenían dificultades para estar en contacto continuo con los procesos de formación, por problemas de desplazamiento al centro donde se imparten los cursos, por escasez de tiempo o por incapacidad física para asistir a clase, tienen ahora un abanico de posibilidad a su disposición.

Se puede decir que el e-learning es la modalidad de educación a distancia, donde tanto el profesor como el alumno hacen uso de medios electrónicos para llevar a cabo el proceso de enseñanza-aprendizaje.

El E-learning se constituye en un escenario de formación donde el aprendizaje, el conocimiento se construye en un entorno de colaboración importante. Esa colaboración tiene lugar gracias a diferentes herramientas de interacción o interactividad, siendo las más comunes los foros, los archivos y documentos compartidos, el chat, las videoconferencias, los wikis y blog entre otros.

Todos los elementos arriba mencionados propician la comunicación, la interacción que mantienen el flujo de información entre los estudiantes y docentes en el proceso formativo. Pero no se debe confundir interacción con interactividad. No es lo mismo, la relación entre seres humanos (interacción) con la participación con una máquina (interactividad).

La interactividad consiste en involucrar al alumno en el proceso y no en colocar diferentes elementos multimedia para que escuche o lea, ya que eso no constituye ninguna interacción. La interacción conlleva necesariamente una práctica con feedback y una orientación al alumnado. Cuanto más manipule los contenidos, más aprenderá. Para ello, es clave que explore, responda cuestionarios autoevaluativos, escuche audios, interactúe con videojuegos y navegue los diferentes enlaces; es decir, actúe e interactué con todos los recursos disponibles. Al respecto Siemens (2014) subraya que uno de los requisitos importantes en el aprendi- zaje en línea es el desarrollo de cursos interactivos y atractivos, actividades que permitan la colaboración estructurada entre pares y plazos flexibles. De acuerdo con Darabi et al. (2013) el mayor impacto son los diseños pedagógicos que incluyen la interacción con los alumnos, la colaboración del estudiante y el seguimiento continuo.

\section{M-Learning}

Esta modalidad surge ante la evolución de los dispositivos móviles como son computadora portátil, tabletas y celulares, al respecto Quinn (2000) considera al Mobile Learning como una extensión del E-learning, pero caracterizado por su independencia respecto a la ubicación en espacio y tiempo. Por su parte, Harris (2001) lo define como una experiencia de aprendizaje en cualquier momento y en cualquier lugar. De acuerdo con Brazuelo \& Gallego (2011), el Mobile Learning desarrolla la construcción del conocimiento, el aprendizaje autónomo y ubicuo gracias a la mediación de dispositivos móviles. Para Motteram (2013) los dispositivos móviles tienen gran influencia en el aprendizaje, por su potencial multimedia y comunicación instantánea en cualquier lugar y hora. Al respecto Ally (2009) argumenta que el aprendizaje a través de la modalidad M-learning tiene la gran ventaja de ser ubicuo, esto tiene un gran impacto significativo en la manera en que los estudiantes aprenden y los docentes los acompañan sin problemas de horario. La UNESCO (2013) refiere que el aprendizaje móvil facilita el acceso a los recursos pedagógicos, permite crear contenidos dentro y fuera del aula, y actualmente constituye una estrategia de esfuerzo para lograr metas educativas de manera eficaz en varios sistemas educativos.

Ejemplos de estas experiencias son mencionados en el informe SCOPEO (2011) Observatorio de la Formación en Red, en el que indica que esta modalidad ofrece momentos irrepetibles para debatir, reflexionar, además aumenta la confianza y la autoestima.

Sin embargo, los elementos a tomar en cuenta según Maceiras, et al (2010) son:

- Definir la estrategia de acuerdo con el tamaño del dispositivo. 
M-learning en el CELEX-ESIA Z. Estrategia educativa que incorpora el WhatsApp al E-learning para el desarrollo de la competencia oral y escrita del idioma inglés

- Los materiales no deben ser de gran extensión, ya que el móvil se consulta en cualquier lugar y las condiciones para la concentración son bajas.

Por todo lo anterior, los mensajes enviados a los alumnos del CELEX son cortos, los recursos utilizados como videos son de máximo dos minutos y las imágenes son de formato pequeño. Esto facilita la consulta rápida y promueve el diálogo entre los participantes.

\section{Competencia oral y escrita del idioma inglés}

Hasta 1950 el método más aceptado de enseñanza de idiomas estaba focalizado en la escritura y la lectura. De acuerdo con Marchisio (2004) más tarde surge la necesidad de mejorar la calidad de la enseñanza del lenguaje que facilitara el aprendizaje, y se modifica la metodología que centra su objetivo en el desarrollo de las habilidades comprensivas y en el manejo del lenguaje oral. Paralelo a ello, surge la concepción mecánica del aprendizaje de la lengua, poniendo un mayor énfasis en la relación estímulo-respuesta. Para la década de los setenta se trató de dar preponderancia a la variable afectiva planteada con aplicación de ideas propuestas a través de la psicología humanista que sostiene que los sentimientos de los alumnos son tan importantes como sus habilidades mentales o cognitivas. Si los estudiantes se muestran hostiles al objeto de estudio, los materiales o los métodos de enseñanza, no tendrán éxito al aprender. Harmer (1993) cita, entre otros, al psicólogo Carl Rogers, quien propone: el alumno debe estudiar lo relevante, debe experimentar al aprender y no ser meramente enseñado, además de tomar en cuenta sus emociones para el proceso de aprendizaje.

Marchisio (2004) refiere que el enfoque humanista busca fortalecer la acción lingüística interpersonal y dar énfasis al desarrollo de las habilidades comunicativas. Además, se incorpora el elemento cultural a la enseńanza del idioma como una segunda lengua, ya que el entorno cultural del alumno es parte de él mismo y su realidad. La explosión tecnológica, el concepto de globalización, y la crisis económica crearon la necesidad de aprender otros idiomas, además la urgencia de cambio en las prácticas educativas; unido a esto, se da el desarrollo tecnológico y el impacto de Internet que hizo posible el contacto con otras culturas con material de estudio actualizado, y la computadora se volvió una herramienta clave.

Durante este milenio, la enseñanza-aprendizaje de los idiomas comenzó a sufrir cambios en su metodología; esto se debe en parte, a la comunicación de manera síncrona o asíncrona $y$, en consecuencia, a la posibilidad de realizar tareas reales en la clase, desplazando la simulación como desempeño de roles, trabajo de a pares, ya que las telecomunicaciones facilitan el aprendizaje sustentado bajo el enfoque de aprendizaje por tareas y situado en el mundo.

Sin embargo, en los estudios realizados por Martín et al. (2014) se menciona que faltan propuestas serias, coherentes y basadas en investigación previa propia del campo de la enseńanza y aprendizaje de segundas lenguas y proponen un marco lingüístico para el desarrollo de aplicaciones, a esto se unen Byrne \& Diem (2014) al indicar que los móviles son cada vez más comunes pero la metodología para la enseñanza de idiomas se encuentra en la fase de "emergencia".

\section{WhatsApp en Educación}

El WhatsApp es una de las herramientas más utilizadas dentro de las redes sociales, por su versatilidad en las comunicaciones; en el ámbito educativo facilita la interacción en actividades de la vida real, al promover el contacto entre estudiantes y profesores; fomentar la interacción entre estudiantes y promover la cooperación académica; motivar el aprendizaje activo; proporcionando un feed-back instantáneo; y desarrolla altas expectativas para un proceso de aprendizaje más significativo (Desai \& Graves, 2006; Farmer, 2003; Rambe \& Bere, 2013).

Actualmente existen diversas experiencias que demuestran la efectividad de este dispositivo, como es el caso de Muhammed, (2014) estudió las actitudes de efectividad del aprendizaje de lenguas a través de móviles para el desarrollo de la habilidad de escuchar. Los resultados de este estudio indican que el aprendizaje con celulares es una forma efectiva de mejorar la comprensión oral. En este 
contexto Zaki \& Yunus (2015) comentan que éstos contribuyen a un aprendizaje significativo si se diseñan estrategias y métodos de aprendizaje que correspondan al contexto. Samsiah \& Azidah (2013) también destacan otros enfoques relacionados al aprendizaje móvil tales como: i) el aprendizaje mixto, ii) aprendizaje interactivo, iii) aprendizaje experimental, iv) aprendizaje basado en problemas.

Mosqueda (2018), profesora adjunta en la Universidad Internacional de La Rioja, publicó en el Blog de la red educación y TIC (Tiching) la entrada titulada: Enganchados al WhatsApp: aprovéchalo como herramienta educativa, donde enuncia las ventajas de utilizar esta aplicación:

- Responder dudas o hacer tutorías en línea.

- Ofrecer una rápida retroalimentación.

- Adjuntar videos, enlaces, imágenes fijas y móviles.

- Desarrollar el trabajo colaborativo.

- Facilitar la aplicación de examénes orales.

- Promover la motivación por el aprendizaje del idioma inglés.

Diversos investigadores sostienen que en esta herramienta de mensajería ubicua altera la manera de escribir al abreviar todo (Bernicot et al., 2014), pero también puede contribuir al desarrollo de los usos lingüísticos en diferentes registros y situaciones, y así, facilitar una mayor competencia lingüística en inglés en el medio digital.

\section{Modelo de Mensajería por Whatsapp Propuesto en el Celex}

El modelo de mensajería por WhatsApp propuesto en el CELEX se sustenta en la integración de los enfoques: constructivista, centrada en el estudiante, aprendizaje colaborativo y aprendizaje informal, unido al uso de todos los recursos de multimedia para el envío de mensajes escritos y hablados en inglés, de una extensión corta y motivacionales por parte de los docentes, utilizando para ello el WhatsApp a través del envío de mensajes visuales, ligas de consulta y aprendizaje social en un ambiente informal, siendo el referente Naismith et al, (2005).
Estrategia aplicada:

1. El profesor da bienvenida al curso

- Comparte el Manual para conocer la plataforma

- Envía el calendario de actividades

- Comenta la forma de trabajar

2. El equipo de sistema se presenta, adjunta el Manual de procedimientos y ofrece sus servicios de apoyo técnico:

- Conexión de micrófonos

- Acceso a la plataforma sobre todo lo que se relaciona con la conexión

- Problemas con las claves para accesar a la plataforma.

- Problemas con la plataforma al momento de hacer evaluaciones, guardar actividades o en los avances a otros niveles

3. En el Nivel 1 el docente en los primeros 15 días acepta mensajes en español, después todo debe ser en inglés. El docente se encarga de hacer las correcciones y enviar material didáctico complementario a la plataforma o solicita el refuerzo de ciertos ejercicios en este espacio virtual.

4. En los demás niveles, no se puede ni escribir o enviar mensajes grabados en español.

5. Todos los días el docente envía mensajes con la siguiente estructura:

- En la mańana saluda aplicando una forma diferente de hacerlo y en caso de ser una fecha en especial, aprovecha para felicitarlos o recordarles la importancia de ese día.

- En la tarde envía mensaje complementario acerca de los ejercicios que deben realizar en plataforma, les recuerda los cierres de actividades y los exámenes a realizar. Envía información complementaria a través de videos cortos o infografía que son debidamente seleccionadas de los llamados recursos abiertos.

- En la noche envía mensajes para concluir el día, además les recuerda la fecha de la videoconferencia y comparte la agenda que se trabajará ese día.

6. Encuentro síncrono se realiza a través de videoconferencia y se utiliza la herramienta de Hangouts. Horas antes se envía la 
M-learning en el CELEX-ESIA Z. Estrategia educativa que incorpora el WhatsApp al E-learning para el desarrollo de la competencia oral y escrita del idioma inglés

invitación para la conexión, así como la agenda a trabajar, cuando la sesión inicia y no se conectan, el docente envía mensajes por medio del WhatsApp de recordatorio.

7. En los cierres de actividades, les envía mensajes recordando que tienen un día o están a horas de concluir con todas las actividades de la plataforma.

8. Las calificaciones se entregan de manera individual, pero se avisa a través del WhatsApp que ya se cuenta con las calificaciones finales.

Cabe señalar, que las dos modalidades comparten el mismo plan de estudios, la misma metodología basada en el enfoque comunicativo para la enseñanza de las lenguas extranjeras, enfatizando la interacción entre pares y con el profesor, valiéndose de las aplicaciones que incluye Cambridge University Press en su plataforma para el desarrollo del programa Touchstone, con ejercicios activos e inductivos centrados en el alumno que los incentiva a trabajar en forma independiente.

La plataforma para los estudiantes motivo del estudio pertenece a Cambridge University Press, que cumple con todas estas características de un entorno educativo, además, propicia la integración de innovaciones como videojuegos, programa para grabar audios y realizar autoevaluaciones.

Todos los ejercicios tienen la opción de realizarse a través de dispositivos móviles, excepto los exámenes finales de cada módulo, que deben responder utilizando la computadora, como una medida de seguridad. Adicional a lo anterior, deben colocar su fotografía y los avances se comparan con el desempeño en las sesiones síncronas.

\section{Materiales Y Métodos}

\section{Participantes}

El estudio se realizó en CELEX de la Escuela Superior de Ingeniería y Arquitectura, de la Unidad Zacatenco, del Instituto Politécnico Nacional. La muestra fue de tipo no probabilística, al ser grupos intactos, conformada por 83 estudiantes, inscritos de febrero a octubre de 2017, de los cuales,
37 siguieron la modalidad presencial de intermedio nivel 5 y 46 de la modalidad virtual de intermedio Nivel 5. El 54\% fueron mujeres y el 46\% hombres. Las edades varían entre 18 a 66 años, porque no todos son egresados de ESIA, sino que había estudiantes matriculados de otras escuelas externas y del IPN.

\section{Instrumento}

El instrumento que se utilizó para medir la comprensión lectora y auditiva fue una adaptación de los exámenes estandarizados de inglés de la Universidad de Cambridge, diseñados para medir las cuatro habilidades lingüísticas del idioma: comprensión auditiva, comprensión lectora, producción oral y escrita, con diferentes niveles de complejidad y distintos propósitos. La adaptación fue realizada por la Coordinación del CELEX-ESIA $Z$, en colaboración con su equipo docente, (Anexo 1). Teniendo una valoración de: Puntaje máximo 51 como óptimo, 45 como medio y 40 como bajo. En la tabla 2 se describe las tres partes que incluye la prueba.

Tabla 2 Respuestas Foro Módulo 1

\begin{tabular}{|c|c|c|}
\hline Part 1 & Part 2 & Part 3 \\
\hline $\begin{array}{l}\text { - Read the } \\
\text { text and } \\
\text { questions } \\
\text { below. } \\
\text { - } 5 \text { preguntas } \\
\text { de opción } \\
\text { múltiple }\end{array}$ & $\begin{array}{l}\text { - Look at the senten- } \\
\text { ces below about } \\
\text { a girl called Anna } \\
\text { and her new school } \\
\text { building. } \\
\text { - Read the text and } \\
\text { decide if each sen- } \\
\text { tence is correct or } \\
\text { incorrect. If it is co- } \\
\text { rrect, mark C and } \\
\text { if it is not correct, } \\
\text { mark I. } \\
\text { - } 6 \text { preguntas. }\end{array}$ & $\begin{array}{l}\text { - Read the text } \\
\text { below and } \\
\text { choose the co- } \\
\text { rrect word for } \\
\text { each space. } \\
\text { - For each ques- } \\
\text { tion, mark the } \\
\text { correct letter } A \text {, } \\
\text { B, C or D. } \\
\text { - } 10 \text { preguntas }\end{array}$ \\
\hline
\end{tabular}

Fuente: Elaboración propia

\section{Tipo y diseño}

El presente estudio cuasiexperimental estableció una medición previa a la intervención y otra posterior. Incluyó un grupo de comparación que no recibió la intervención y que se evalúa también antes y después con el fin de medir otras variables 
externas que cambian el efecto esperado por razones distintas a la intervención.

\section{Procedimiento}

El estudio se realizó siguiendo cinco fases.

\section{Fase 1: Diagnóstico preliminar}

Antes de iniciar el estudio para poder diseñar la estrategia, se evaluaron a los estudiantes de las modalidades presencial y virtual de inglés intermedio Nivel cinco, con el mismo instrumento. Al comparar los resultados, se observó menor promedio en la expresión oral y escrita en los estudiantes virtuales. Con la finalidad de corroborar los resultados, se realizaron conversaciones con los docentes para verificar si los estudiantes de la modalidad virtual realizaban las actividades de la plataforma para el desarrollo de las competencias donde salían con bajo puntaje. Después se procedió a revisar si los estudiantes realizaban todas las actividades y se comprobó que no podían pasar de nivel hasta concluir de manera exitosa todo. Frente a esto, se buscaron estrategias alternas a la plataforma para corregir la pronunciación de manera personalizada, así como la competencia escrita.

\section{Fase 2: Selección de la estrategia a utilizar}

Con los datos de la Fase 1, se decidió buscar una alternativa adicional a la modalidad E-learning, como es el M-learning a través de la mensajería del WhatsApp para elevar las competencias comunicativas oral y escrita, considerando el fundamento teórico que sustenta poder utilizar este medio como estrategia de aprendizaje.

\section{Fase 3: Implementación de Mensajeria WhatsApp}

Esta fase consistió en la implementación del uso de la aplicación del WhatsApp, previamente se utilizó una encuesta para saber si todos los estudiantes contaban con un celular de los llamados inteligentes y después se procedió a la formación de grupos por nivel.

\section{Fase 4: Aplicación del uso de WhatsApp}

En esta fase se puso en marcha la estrategia del uso del WhatsApp, en la cual el profesor sigue las estrategias propuestas por la Coordinación, como son:

- Promover la participación de todos los estudiantes.

- Fomentar el aprendizaje independiente.

- Retroalimentar de forma instantánea

- Motivar de manera constante a los estudiantes a concluir las actividades.

- Ser empático y utilizar todo tipo de multimedia como son Gif, imágenes y videos (recursos abiertos).

- Compartir información complementaria tomando en cuenta el perfil de los estudiantes y los avances. (Algunos mensajes se pueden ver en el Anexo 2)

\section{Fase 5: Análisis de los resultados.}

Se procedió a analizar los resultados del pretest y postest de la modalidad virtual, a través de la estadística inferencial, utilizando el SPSS.

\section{Resultados}

A continuación, se muestran los resultados del pretest y postest aplicado a la modalidad virtual y presencial, en el cual se comprueba un bajo puntaje en la competencia oral que aplica el docente de manera directa fuera de plataforma y es ligeramente menor que la escrita, los resultados en extenso se encuetran en el apartado del Anexo 3, tablas 4, 5, 6 y 7.

En esta investigación se utilizó la prueba $\mathrm{t}$ - Student para muestras relacionados, el cual permitió comparar al grupo experimental y de control, utilizando un pretest y postest. La diferencia estadística del uso de Whatspp entre el antes y después fue significativa en TW, List, TList y final ( $\mathrm{p}<0.05)$, comparada con el grupo control en donde se encontró diferencia estadística en final $(\mathrm{p}<0.05)$, como se observa en la tabla 3. 
M-learning en el CELEX-ESIA Z. Estrategia educativa que incorpora el WhatsApp al E-learning para el desarrollo de la competencia oral y escrita del idioma inglés

Tabla 3. Promedios de calificación por área de conocimiento en ambos grupos y nivel de significancia intragrupos.

\begin{tabular}{|c|c|c|c|c|c|c|c|c|c|c|}
\hline & & Read & UofE & $\begin{array}{l}T L / U \\
\text { of } E\end{array}$ & Wr & $\mathrm{Wr}$ & TW & List & T List & Final \\
\hline \multirow{3}{*}{$\begin{array}{l}\text { Contr } \\
N=37\end{array}$} & $\bar{x}$ PRE & 9.67 & 7.04 & 64.78 & 1.48 & 4.11 & 49.63 & 10.78 & 45.74 & 68.04 \\
\hline & $\bar{x}$ POST & 10.07 & 7.07 & 68.59 & 1.63 & 4.07 & 57.04 & 12.04 & 52.22 & 81.26 \\
\hline & T-S & 0.622 & 0.928 & 0.3474 & 0.672 & 0.843 & 0.140 & 0.383 & 0.339 & $<0.05$ \\
\hline \multirow{3}{*}{$\begin{array}{l}\text { Exper } \\
\mathrm{N}=45\end{array}$} & $\bar{x}$ PRE & 10.23 & 7.03 & 68.74 & 1.57 & 4.11 & 56.57 & 5.06 & 44.91 & 61.54 \\
\hline & $\bar{x}$ POST & 10.23 & 7.03 & 70.74 & 1.57 & 4.11 & 76.74 & 13.57 & 82.46 & 87.31 \\
\hline & T-S & 1.0 & 1.0 & 0.6020 & 1.0 & 1.0 & $<0.05$ & $<0.05$ & $<0.05$ & $<0.05$ \\
\hline
\end{tabular}

$\mathrm{P}=$ promedio, $\mathrm{T}-\mathrm{S}=$ Prueba T-Student

\section{Discusión y Conclusiones}

De todos los avances tecnológicos el celular se considera como el de mayor aceptación por su facilidad de uso, conectividad y esto lo está convirtiendo en un aliado para la educación, de acuerdo Ally (2009) el aprendziaje a través de la modalidad M-learning tiene la gran ventaja de ser ubicuo, esto permite a los estudiantes aprender en todo lugar y a cualquier hora.

La presente investigación se basó en los principios de Naismith et al, (2005), para desarrollar la competencia oral y escrita, aprovechando una de las tecnologías más utilizadas por los estudiantes, lo que les permitió de manera informal, comunicarse todos los días en el idioma inglés.

Por otra parte, los resultados arrojados en el postest aplicado a los estudiantes de la modalidad virtual, muestran un avance significativo en la expresión escrita y oral con respecto al pretest. Con esto es posible comprobar que la integración del M-learning a través del uso de WhatsApp a la modalidad E-learning, es viable al permitir a los estudiantes convivir con el idioma inglés de una manera cotidiana, al estar en contacto constante con los mensajes del docente además de realizar una serie de actividades de manera flexible sin importar el tiempo y el lugar.

El WhatsApp además favoreció la interacción entre estudiantes y docentes de una manera cotidiana, al registrarse un promedio diario de 20 a 30 mensajes, desde el saludo en la mańana, la res- puesta inmediata de los estudiantes, además del envío de material complementario, recordatorio de actividades a realizar, agenda de temas a tratar en las videoconferencias, hasta la publicación de los resultados de las evaluaciones, y el acompañamiento ante el surgimiento de problemas técnicos.

La aplicación de esta estragia también:

- Favoreció el trabajo colaborativo y el aprendizaje social.

- Se fomentó la interacción social.

- Se estableció una línea de comunicación directa: docente estudiante, estudiante-estudiante y la respuesta inmediata en cuanto a dudas.

- Contributó a desarrollar la competencia oral al grabar mensajes de audio y escritos en el idioma inglés.

Además de elevar su promedio en la competencia oral y escrita los estudiantes de la modalidad virtual mostraron gran satisfacción con el uso de mensajería como estrategia, al permitir una comunicación constante con su docente y compañeros. La herramienta del WhatsApp fortalece el modelo educativo "centrado en el estudiante", al dar seguimiento uno a uno, lo que no se lograba con la modalidad E-learning.

Este trabajo abre nuevas líneas de investigación como es seguir investigando sobre la satisfacción 
de los estudiantes, la motivación es otro elemento que permitirá identificar las razones porque la mayoría de los estudiantes que inician concluyen todos los ciclos. Otro elemento que no se ha considerado es el análisis de datos de comportamiento que arroja la plataforma, para determinar qué actividades resultan de mayor complejidad, mayor reprobación, identificar estilos de aprendizaje predominante y tiempo invertido real en plataforma, todo esto, para encaminar el aprendizaje individualizado. En lo que respecta a los cursos de la modalidad presencial, siguen sin modificar su estructura.

\section{REFERENCIAS BIBLIOGRÁFICAS}

Muhammed, A. (2014). The impact of mobiles on language learning on the part of English foreign language (EFL) university students. Procedia-Social and Behavioral Sciences, 136, 104-108. http://doi.org/10.1016/j.sbspro.2014.05.297

Ally, M. (2009). Mobile learning: Transforming the delivery of education and training. Athabasca University Press. Recuperado de http://citeseerx.ist.psu.edu/viewdoc/download?doi=10.1.1.475.2163\&rep=rep1\&type=pdf

Ally, M., \& Samaka, M. (2013, February). Improving Communication skills in the workplace using mobile Learning. Presentation at the Symposium on Mobile Learning, UNESCO Mobile Learning Week, Paris, France.

Baird, W., Secrest, J., Padgett, C., Johnson, W., \& Hagrelius, C. (2016). Smartphones and Time Zones. The Physics Teacher, 54 (6). https://doi.org/10.1119/1.4961177

Bernicot, J., Goumi, A., Bert-Erboul, A. \& Volckaert-Legrier, O. (2014). How do skilled and less-skilled spellers write text messages? A longitudinal study. Journal of Computer Assisted Learning. https://doi.org/10.1111/jcal.12064

Brazuelo, f. \& Gallego, D. (2011) Mobile Learning. Los dispositivos móviles como recurso educativo. Editorial MAD, S.L., Sevilla, Espańa.

Byrne, J., \& Diem, R. (2014). Profiling Mobile English Learners. The Jalt CALL Journal, 10 (1), 3-19. Recuperado de https://files.eric.ed.gov/fulltext/EJ1107975.pdf

Chevrier, J., Madani, L., Ledenmat, S., \& Bsiesy, A. (2013). Teaching classical mechanics using smartphones. The Physics Teacher, 51 (6). https://doi.org/10.1119/1.4818381

Darabi, A., Liang, X., Suryavanshi, R., \& Yurekli, H. (2013). Effectiveness of online discussion strategies: A meta-analysis. American Journal of Distance Education, 27 (4), 228-241. http://dx.doi.org/10.1080/08923647.2013.837651

Desai, C. \& Graves, S. (2006). Instruction via Instant Messaging Reference: What's Happening? The
Electronic Library, 24 (2), 174-189. https://doi. org/10.1108/02640470610660369

Farmer, R. (2003). Instant Messaging - Collaborative Tool or Educator's nightmare! The North American Web-Based Learning Conference (nAWeb 2003). Recuperado de https://www.student.cs.uwaterloo.ca/ - cs492/10public_html/ papers/im.pdf

Forinash, K., \& Wisman, R. (2015). Photogate Timing with a Smartphone. The Physics Teacher, 53 (4). https://doi. org/10.1119/ 1.4914566

Kuhn, J., \& Vogt, P. (2013). Analyzing Acoustic Phenomena with a Smartphone Microphone. The Physics Teacher, 51 (2). https://doi.org/10.1119/1.4775539

Hall, J. (2013). More smartphone acceleration. The Physics Teacher, 51 (6). https://doi.org/10.1119/1.4772022

Harmer, J. (1993). The Practice of English Language Teaching. New york: Longman.

Harris, P. (2001). Going mobile. Learning Circuits. American Society for Training and Development. Magazine All about eLearning 2001.

International Telecommunication Union. (2016). Measuring the Information. Society Report. Geneva, Switzerland: International Telecommunication Union. Recuperado de https://www.itu.int/en/ITU-D/Statistics/Documents/publications/misr2016/MISR2016-w4.pdf

Maceiras, R., Cancela, A., Sánchez, A., Casar, A. \& Urrejola, S. (2010). Adaptation of a Virtual Campus for Mobile Learning Devices. Recuperado de http://www.psut.edu.jo/ sites/EDUCON/program/contribution1153_b.pdf

MacIsaac, D. (2015). Smartphones in a guitar redux. The Physics Teacher, 53 (3). https://doi.org/10.1119/1.4908097

Martín, E., Arús-Hita, J., Rodríguez, P., \& Calle-Martínez, C. (2014). Reall: Rubric for the evaluation of apps in language learning, En F. Navaridas, R. Santiago, J. Sota Eguizabal, M., Jiménez, T. y González, L. (Eds.), Actas de las Jornadas Internacionales Tecnología Móvil e Innovación en el Aula: Nuevos Retos y Realidades Educativas.

Mau, S., Insulla, F., Pickens, E., Ding, Z., \& Dudley, S. (2016). Locating a smartphone's accelerometer. The Physics Teacher, 54 (4). https://doi.org/10.1119/1.4944372

Marchisio, P. (2004). La enseñanza del inglés a distancia. Primer Congreso Virtual Latinoamericano de Educación a Distancia. Documento impreso.

Motteram, G. (2013). Innovations in learning technologies for English Language teaching. UK: British Council.

Moreno, J. (2013). ¿Cómo implementar el e-learning en los procesos de enseńanza-aprendizaje? Observatorio de Tecnológico, Ministerio de Educación, Cultura y Deporte. Gobierno de España. Recuperado de http://recursostic. educacion.es/observatorio/web/ca/internet/recursos-online/1089-icomo-implementar-el-e-learning-en-los-procesos-de-ensenanza-aprendizaje

Monteiro, M., Vogt, P., Stari, C., Cabeza, C., \& Marti, A. (2016). Exploring the atmosphere using smartphones. The 
M-learning en el CELEX-ESIA Z. Estrategia educativa que incorpora el WhatsApp al E-learning para el desarrollo de la competencia oral y escrita del idioma inglés

Physics Teacher, 54 (5). https://doi.org/10.1119/1.4947163

MotorolaBlog (2018, February 21). Motorola Study Shows Alarming Result That Confirm Need for Better Phone-life Balance. Recuperado de https://blog.motorola.com/2018/02/21/motorola-study-shows-alarming-results-that-confirm-need-for-better-phone-life-balance/

Mosqueda, I. (2018). Enganchados en el WhatsApp: aprovéchalo como herramienta educativa. Recuperado de http:// blog.tiching.com/enganchados-WhatsApp-aprovechalo-como-herramienta-educativa/

Motteram, G. (2013). Innovations in learning technologies for English Language teaching. Londres: British Council. Recuperado de https://www.teachingenglish.org.uk/sites/ teacheng/files/C607\%20Information\%20and\%20Communication_WEB\%20ONLY_FINAL.pdf

Müller, A., Vogt, P., Kuhn, J., \& Müller, M. (2015). Cracking knuckles - A smartphone inquiry on bioacoustics. The Physics Teacher, 53 (5). https://doi.org/10.1119/1.4917442

Naismith, L., Lonsdale, P., Vavoula, G. \& Sharples, M. (2005) Literature Review in Mobile Technologies and Learning. A Report for NESTA Futurelab. Recuperado de https://www.nfer.ac.uk/publications/FUTL15/FUTL15.pdf

Primer Encuesta Nacional sobre consumo digital y cultura (2015). Coordinado por Consultores de Investigación y Comunicación. México. IBBY México, Banamex. Recuperado de http://www.ibbymexico.org.mx/images/ENCUESTA_ DIGITAL_LECTURA.pdf

Quinn, C. (2000) "M-Learning: Mobile, Wireless, in Your-Pocket Learning”. LiNE Zine. Fall. Recuperado de http://www.linezine.com/2.1/features/cqmmwiyp.htm

Rambe, P., \& Bere, A. (2013). Using Mobile instant Messaging to Leverage Learner Participation and Transform Pedagogy at a South African University of Technology. British Journal of Educational Technology. British Journal of Educational Technology, 44 (4), 531-672. https://doi. org/10.1111/bjet.12057

Shakur, A., \& Kraft, J. (2016). Measurement of Coriolis Acceleration with a Smartphone. The Physics Teacher, 54 (5). https://doi.org/10.1119/1.4947157

Samsiah, B., \& Azidah, A. (2013). Adoption and application of mobile learning in the education industry. 6th International Conference on University Learning and Teaching (InCULT 2012). Procedia-Social and Behavioral Sciences, 90, 720-729. Htpps://doi.org/10.1016/j.sbspro.2013.07.145

SCOPEO (2011). Aproximación pedagógica a las plataformas open source en la universidad española, marzo de 2011. Monográfico SCOPEO, nº 2. Recuperado de http://scopeo. usal.es/images/documentoscopeo/scopeo_monografico002. pdf

Siemens, G. (2014). Digital Learning Research Network. Learnspace, November. Recuperado de http://www.elearnspace.org/blog/201 4/11/18/digital-learning-research- network-dlrn/

Tornaría, F., Monteiro, M., \& Marti, A. (2014). Understan- ding coffee spills using a smartphone. The Physics Teacher, 52 (8). https://doi.org/10.1119/1.4897595

Vogt, P., Kuhn, J., \& Neuschwander, D. (2014). Determining ball velocities with smartphones. The Physics Teacher, 52 (6). https://doi.org/10.1119/1.4893100

UNESCO (2013) El futuro del aprendizaje móvil. Implicaciones para la planificación y formulación de políticas. París, UNESCO. Recuperado de http://unesdoc.unesco.org/images/0021/002196/219637s.pdf

Zaki, A., \& Yunus, M. (2015). Potential of Mobile Learning in Teaching of ESL Academic Writing. English Language Teaching, 8(6). Recuperado de http://www.ccsenet. org/journal/index.php/elt/article/view/49403

\section{Anexo 1}

PET (Preliminary English Test for Schools)

PAPER 1: READING AND WRITING

READING

\section{Part 1. Read the text and questions below.}

\section{JAZZ}

By Katie Atkins, aged 14

I play the trumpet in my school jazz band. Last month we held a jazz competition with bands from local high schools and our band won!

Each band had their own costumes, ranging from black school uniforms like my band wore, to brightly-coloured T-shirts. We didn't look much like adult professional bands, but all of us were used to performing in competitions, so the quality of playing was amazing, especially considering everyone was so young. Players from each band even created new tunes right there on stage. It was exciting to watch - but even better when my band played on stage!

We have a great jazz band at my school, but not everyone who wants to play in it gets accepted only about half, in fact. But anyone who's keen to play goes to jazz practice before school, and we 
often spend time together after school, listening to jazz and learning its language. There are also trips to jazz summer camps across the country I've been to a couple and learnt a lot.

Adults are often surprised that young people are getting interested in jazz. My music teacher thinks it's because pop music isn't challenging enough for people like me who are serious about music. But I find it exciting because it's both new and old at the same time - you can create your own music, but you also feel you're part of its history, as you're playing on stage in the same way as great jazz performers before you.

My school's really lucky because we have great teachers, and parents who've supported us all the way. Without them, we'd never get anywhere with our music!

For each question, mark the correct letter A, B, C or D

1. What is Katie trying to do in the text?
A Encourage young people to try to listen to more jazz.
B Explain how jazz is becoming popular with young people.
C Advertise young people's jazz events in her area.
D Give advice on how to create great jazz music.

2. Katie says the bands in her school's jazz competition
A played music they'd written themselves before they came.
B had little experience of playing in public.
C played at a high level despite their age.
$\mathrm{D}$ were dressed to look like professional jazz bands.

3. Most people at Katie's school who are interested in jazz
A attend early jazz sessions at school.
B join the school jazz band.
C learn about jazz in after-school classes.
D go to jazz summer camps at the school.

4. Why does Katie enjoy playing jazz so much?
A she finds it easier to learn than other for- ms of music.
B she thinks it is more serious than pop music.
C she likes the chance to perform with others on stage.
D she feels in touch with jazz players of the past.

5. Which of the following would Katie write to a friend?

A The competition was great, but I think I preferred being in the audience to playing - I was nervous!

B My mum and dad always do all they can to help with my trumpet playing - I couldn't do this successfully without their help.

C One band wore really colourful clothes, but we chose dark costumes. maybe that's one reason why they won instead of us.

D One music teacher left a while ago and no one's replaced him yet. We just haven't got anyone good to help.

\section{Part 2: Look at the sentences below about a girl called Anna and her new school building. Read the text and decide if each sentence is correct or incorrect. If it is correct, mark $C$ and if it is not correct, mark I.}

6. Anna's school is the first one in her country to be made completely ofwood.

$$
\text { C I }
$$

7. Anna had mixed feelings about seeing the space cleared ready for building the school. C I

8. Builders managed to carry on with work on the school despite the weather.

C I

9. There is one room that is kept just for local people to use for meetings.

C I

10. The children find the new changing roomscon- 
M-learning en el CELEX-ESIA Z. Estrategia educativa que incorpora el WhatsApp al E-learning para el desarrollo de la competencia oral y escrita del idioma inglés

venient when they do sports on their field. C I

11. The inside of Anna's school depends on the people and machines in it to be warm. C I

12. The new school building has great benefits for people who like to sing.

C I

13. The builders wanted to do something traditional for the school when they'd finished the roof. C I

14. Pictures of the wind turbine behind the school can now be seen online.

C I

15. Thepupilsarebusypreparingtodoaperformancefor their parents in their new schoolbuilding. C I

\section{ANNA'S NEW SCHOOL}

It's been a very exciting week, as we've just moved into our new school building! It's an amazing place, as the builders have used wood for the whole thing, just like in our old school, but it's also one of the first in my country designed to be really environmentally friendly.

The school took months to build, so we saw it all happening. It was really exciting seeing the builders clear the space where it would be, although it also meant that some lovely

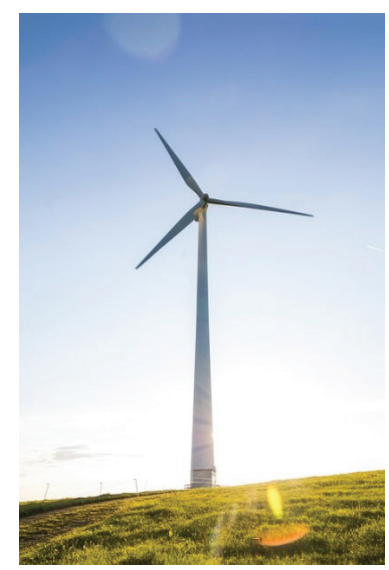
open land disappeared.

And the noise of the building machines was really loud, although the builders often had to stop work because it rained so hard. We all began to think the building would never be finished.

We have five classrooms now, which are enormous - much bigger than in our previous school next door, which was very old. We share one room with people in the town when they want to have meetings and so on. There's a nursery too, a lovely dining room, and really big changing rooms which we're looking forward to using as soon as our new sports field is ready.

When you come into the school, one of the first things you notice is that there aren't any heaters in the building! Instead, the heat for the school comes from all of us inside it - the children, the teachers and the computers we use. The building is so well designed that it holds all the heat inside - and all the sound too, so if we sing inside our classrooms, we can almost believe we're inside a concert hall!

Another thing you'll notice is that the school roof has a tree on top of it! It was put there by the builders once they'd finished the roof, because they said it was the custom in Austria, the country where the roof was made. The electricity for lights and computers comes from a wind turbine on a hill behind the school. We went with our teacher to look at it yesterday, and it goes round really fast. It's so big, it can be seen for miles! We took some pictures which we're planning to put on the website.

Even though we're already using the school building, there are still some parts to be added to it - for example, at the moment we're putting on a play for parents to come and see, but we still have to walk back to our old school hall to do it. The replacement will be ready by next year, though. We're so pleased with our new school, and our teachers say we'll all learn much faster now we're in it!

\section{Part 3. Read the text below and choose the co- rrect word for each space. For each question, mark the correct letter $A, B, C$ or $D$.}

Example:

0 A one $\mathrm{B}$ some $\mathrm{C}$ any $\mathrm{D}$ many

Answer B

\section{SHARKS}

Sharks are (0) of the most frightening creatures in our oceans. They are well pre- 
pared for feeding under water because they can (16) very well, and they can also

(17) movement through special lines on the sides of their bodies. These make sharks very (18) that become their food. for smaller sea creatures

Although sharks are similar (19) other fish in a number of ways, their bodies are different. For example, unlike other fish, most sharks (20) to swim all the time in order to breathe and stay alive, (21)

they hardly sleep at all. Also, if sharks are turned over on their backs, they can stop moving (22) for researchers $(23)$
required to $(24)$ This is a very useful technique are often them to (25) sharks. It allows these fascinating creatures. out more about

16 A watch B look C notice D see

17 A touch B feel

C know

D catch

18 A dangerous

19 A with B from

C to $\mathrm{D}$ of

20 A bought $B$ need

C must D should

21 A so $\mathrm{B}$ as

C but

$\mathrm{D}$ or

22 A perfectly B finally C fully D completely

23 A which B who C what D whose

24 A sort B care C deal D handle

25 A discover B search C find D study WRITING

Part 1: Here are some sentences about a school art exhibition. For each question, complete the second sentence so that it means the same as the first. Use no more than three words. Write only the missing words.

Example: We had an exhibition of our art work in the school hall last night.

There an exhibition of our art work in the school hall last night.
1. Unfortunately we had too many pictures to put on the walls.

Unfortunately we didn't space for all our pictures.

2. The photos in the exhibition were taken by the youngest pupils.

The youngest pupils the photos in the exhibition.

3. I don't think I put my best pictures in the exhibition.

I've produced pictures than the ones I put in the exhibition.

4. There were such a lot of people that i couldn't find my friends.

There were people that I couldn't find my friends.

5. Our parents all said that we should have another exhibition soon.

Ourparentsallsaid 'Why have another exhibition soon?'

\section{Part 2: You have just been to the town centre to} spend some birthday money on a book. Write an email to your english friend, Sam. in your email you should:

- say what kind of book you bought

- say why you chose the book

- suggest when you can show your friend what you bought.

Write 35-45 words.

\section{PAPER 2 LISTENING}

Part 1: You will hear an interview with a writer called Peter Taylor. For each question, choose the correct answer.

1. In Peter's first book, the story takes place in ( )

A a country which he's recently been to.

B a country where he lived as a child.

C the country where he was born. 
2. When Peter first went to England, he visited his ( )
A grandfather.
$\mathrm{B}$ uncle and aunt.
C cousins.

3. Peter spends most of his year ( )
A near the beach.
$\mathrm{B}$ in a city.
C at his farm.

4. What problem did Peter have in the desert? ( )
A His vehicle broke down.
B He didn't have enough water.
C He was frightened by an animal.

5. In his spare time, Peter usually ( )
A goes to the cinema.
$\mathrm{B}$ gets together with friends.
C does photography.

6. What does Peter want to do in the future? ( )
A publish another novel
B write a history book
C spend more time travelling

Part 2: You will hear a radio announcer giving details about a local Motor Show. For each question, fill in the missing information in the spaces. Write no more than three words and/or a number.

\section{WEEKEND ACTIVITIES IN FISHPORT}

Your choice of activities

BIRD ISLAND WALK - 10.00 a.m.

Don't forget to take your (7) with you

\section{GUITAR DAY}

Will be held in the (8) Centre

PLAZA CINEMA - 2.30 p.m.

A programme of (9) films for all the

\section{CYCLE RACE}

This year's route is through the (10)

GREEN STREET THEATRE - 3.00 p.m.

'The Long (11) ................... ' - a play for children

CAMFORD CASTLE - open all day

Display of (12) ................... used in medicine

Part 3: Look at the six sentences for this part. You will hear a conversation between a boy, Oliver, and a girl, Hannah, about a party. Decide if each sentence is correct or incorrect. If it is correct, choose Yes. if it is not correct, choose No. Mark $\square$ in the correct column.

\section{YES NO}

1. Sally knows that Ian is an excellent cook

2. Sally is happy to eat less meat than she used to.

3. Ian learned about cooking by watching other people.

4. Ian and Sally agree that schools should offer more cooking classes

5. Sally is willing to pay more for dishes that are already prepared.

6. Ian suggests that simple recipes are best.

Adapted from Complete Pet for Schools @ Cambridge University Press 2010 this Page may be PhOtOCOPied 821 


\section{Anexo 2}

Mensajes de WhatsApp entre docentes y estudiantes

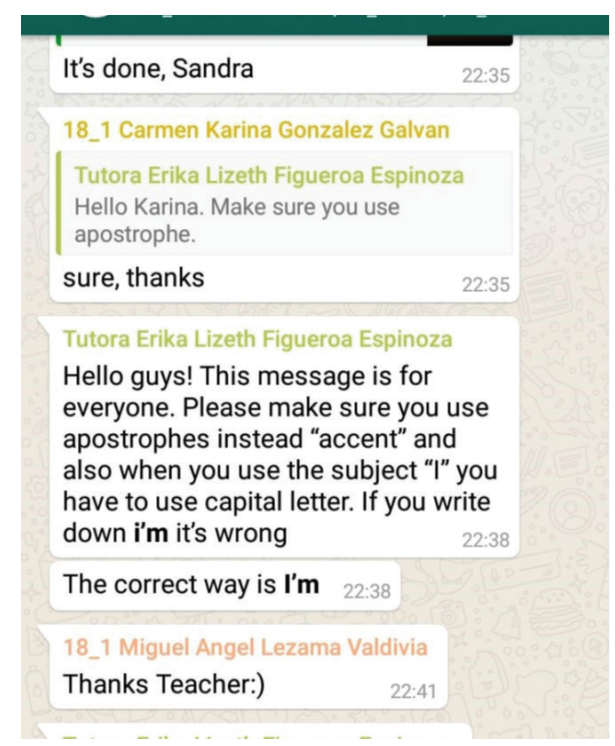

\begin{tabular}{l}
\hline GIF \\
17_2 Nancy Areli Ortiz Diaz \\
Thank you teacher! \\
My g \\
mail is arebuor@gmail.com 15:54 \\
18_1 Jesus Ricardo Licona Muños \\
My gmail is: licricardo1@gmail.com. \\
My video call is 10pm. \\
Tutora Erika Lizeth Figueroa Espinoza \\
Thanks $\downarrow$ 21:00 \\
\hline
\end{tabular}

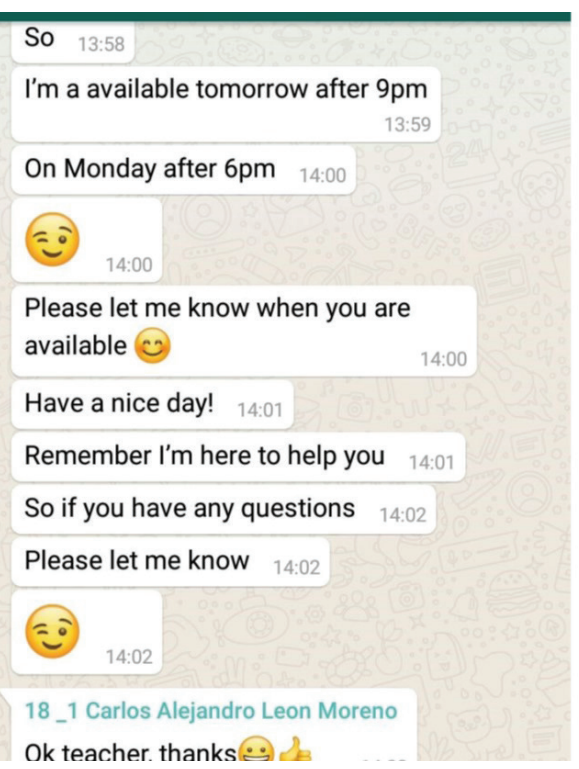

\section{Anexo 3}

Tabla 4. Resultados pretest, grupo. Inglés intermedio nivel 5. Modalidad virtual

\begin{tabular}{|c|c|c|c|c|c|c|c|c|c|}
\hline No. & $\begin{array}{l}\text { Rea- } \\
\text { ding } \\
\text { (15) }\end{array}$ & $\begin{array}{l}\text { Use of } \\
\text { Eng } \\
(10)\end{array}$ & $\begin{array}{l}\text { T L/U } \\
\text { of } E\end{array}$ & $\begin{array}{l}\text { Wr } \\
\text { (5) }\end{array}$ & $\begin{array}{l}\text { Wr } \\
\text { (5) }\end{array}$ & $\begin{array}{l}\text { Total } \\
\text { W }\end{array}$ & $\begin{array}{l}\text { List } \\
(6)\end{array}$ & $\begin{array}{l}\text { Total } \\
\text { List }\end{array}$ & Final \\
\hline 1 & 13 & 7 & 80 & 2 & 5 & 70 & 4 & 70 & 63 \\
\hline 2 & 8 & 6 & 56 & 1 & 4 & 50 & 3 & 45 & 50 \\
\hline 3 & 12 & 7 & 76 & 2 & 4 & 60 & 4 & 65 & 65 \\
\hline 4 & 11 & 7 & 72 & 1 & 4 & 50 & 6 & 50 & 50 \\
\hline 5 & 10 & 4 & 56 & 2 & 4 & 60 & 7 & 65 & 58 \\
\hline 6 & 13 & 8 & 84 & 2 & 5 & 70 & 6 & 80 & 78 \\
\hline 7 & 10 & 5 & 60 & 0 & 3 & 30 & 6 & 30 & 52 \\
\hline 8 & 7 & 9 & 64 & 2 & 5 & 70 & 5 & 80 & 71 \\
\hline 9 & 14 & 8 & 88 & 4 & 4 & 80 & 4 & 80 & 72 \\
\hline 10 & 9 & 7 & 64 & 0 & 4 & 40 & 8 & 40 & 58 \\
\hline 11 & 7 & 7 & 56 & 1 & 4 & 50 & 3 & 60 & 55 \\
\hline 12 & 5 & 4 & 36 & 0 & 5 & 50 & 8 & 60 & 58 \\
\hline 13 & 7 & 6 & 52 & 2 & 4 & 60 & 5 & 50 & 54 \\
\hline 14 & 7 & 8 & 60 & 2 & 3 & 50 & 5 & 25 & 55 \\
\hline 15 & 13 & 10 & 92 & 2 & 4 & 60 & 7 & 85 & 79 \\
\hline 16 & 8 & 7 & 60 & 1 & 3 & 40 & 9 & 45 & 58 \\
\hline 17 & 13 & 9 & 88 & 3 & 4 & 70 & 8 & 90 & 82 \\
\hline 18 & 7 & 7 & 56 & 1 & 4 & 50 & 6 & 60 & 57 \\
\hline 19 & 7 & 5 & 48 & 1 & 4 & 50 & 5 & 50 & 49 \\
\hline 20 & 14 & 9 & 82 & 4 & 5 & 80 & 7 & 75 & 79 \\
\hline 21 & 14 & 9 & 92 & 4 & 5 & 90 & 6 & 30 & 70 \\
\hline 22 & 14 & 5 & 76 & 0 & 3 & 30 & 1 & .5 & 55 \\
\hline 23 & 10 & 7 & 68 & 0 & 5 & 50 & 1 & .5 & 50 \\
\hline 24 & 10 & 7 & 68 & 1 & 4 & 50 & 3 & 15 & 54 \\
\hline 25 & 10 & 6 & 64 & 0 & 4 & 40 & 4 & 20 & 51 \\
\hline 26 & 15 & 10 & 100 & 4 & 4 & 80 & 6 & 30 & 70 \\
\hline 37 & 4 & 7 & 44 & 2 & 3 & 50 & 4 & 20 & 58 \\
\hline 38 & 14 & 5 & 76 & 0 & 3 & 30 & 1 & .5 & 55 \\
\hline 39 & 10 & 7 & 68 & 0 & 5 & 50 & 1 & .5 & 60 \\
\hline 40 & 10 & 7 & 68 & 1 & 4 & 50 & 3 & 15 & 64 \\
\hline 41 & 10 & 6 & 64 & 0 & 4 & 40 & 4 & 20 & 51 \\
\hline 42 & 7 & 7 & 56 & 1 & 4 & 50 & 6 & 60 & 55 \\
\hline 43 & 7 & 5 & 48 & 1 & 4 & 50 & 8 & 50 & 69 \\
\hline 44 & 14 & 9 & 92 & 4 & 5 & 90 & 7 & 75 & 79 \\
\hline 45 & 14 & 9 & 92 & 4 & 5 & 90 & 6 & 30 & 70 \\
\hline
\end{tabular}

Fuente elaboración propia, 2018 
M-learning en el CELEX-ESIA Z. Estrategia educativa que incorpora el WhatsApp al E-learning para el desarrollo de la competencia oral y escrita del idioma inglés

En la tabla 5 se muestran los resultados del examen aplicado a la modalidad presencial.

Tabla 5. Resultados del pretest, grupo 2. Inglés intermedio nivel 5. Modalidad presencial.

\begin{tabular}{|c|c|c|c|c|c|c|c|c|c|}
\hline No. & $\begin{array}{l}\text { Rea- } \\
\text { ding } \\
\text { (15) }\end{array}$ & $\begin{array}{l}\text { Use } \\
\text { of } \\
\text { Eng } \\
(10)\end{array}$ & $\begin{array}{l}\text { T L/U } \\
\text { of } E\end{array}$ & $\begin{array}{l}W r \\
\text { (5) }\end{array}$ & $\begin{array}{l}\mathrm{Wr} \\
(5)\end{array}$ & $\begin{array}{l}\text { Total } \\
\text { W }\end{array}$ & $\begin{array}{l}\text { List } \\
\text { (6) }\end{array}$ & $\begin{array}{l}\text { Total } \\
\text { List }\end{array}$ & Final \\
\hline 1 & 10 & 7 & 70 & 2 & 5 & 60 & 14 & 60 & 60 \\
\hline 2 & 8 & 6 & 50 & 1 & 4 & 45 & 9 & 40 & 65 \\
\hline 3 & 10 & 7 & 56 & 2 & 4 & 40 & 13 & 45 & 62 \\
\hline 4 & 11 & 7 & 72 & 1 & 4 & 40 & 10 & 40 & 55 \\
\hline 5 & 6 & 4 & 50 & 2 & 4 & 50 & 10 & 55 & 63 \\
\hline 6 & 13 & 8 & 64 & 2 & 5 & 00 & 16 & 40 & 52 \\
\hline 7 & 10 & 5 & 60 & 0 & 3 & 25 & 6 & 30 & 60 \\
\hline 8 & 7 & 8 & 60 & 2 & 5 & 70 & 14 & 80 & 76 \\
\hline 9 & 14 & 8 & 88 & 4 & 4 & 80 & 16 & 80 & 72 \\
\hline 10 & 9 & 7 & 64 & 0 & 4 & 40 & 16 & 40 & 70 \\
\hline 11 & 7 & 7 & 56 & 1 & 4 & 40 & 12 & 40 & 70 \\
\hline 12 & 10 & 7 & 68 & 0 & 5 & 50 & 1 & 15 & 70 \\
\hline 13 & 7 & 6 & 52 & 2 & 4 & 50 & 15 & 50 & 60 \\
\hline 14 & 7 & 8 & 60 & 2 & 3 & 50 & 15 & 25 & 70 \\
\hline 15 & 13 & 10 & 92 & 2 & 4 & 60 & 17 & 85 & 81 \\
\hline 16 & 8 & 7 & 60 & 1 & 3 & 40 & 9 & 45 & 80 \\
\hline 17 & 13 & 9 & 88 & 3 & 4 & 70 & 18 & 90 & 72 \\
\hline 18 & 5 & 7 & 56 & 1 & 4 & 50 & 12 & 60 & 60 \\
\hline 19 & 7 & 5 & 48 & 1 & 4 & 50 & 10 & 50 & 70 \\
\hline 20 & 14 & 9 & 82 & 4 & 5 & 90 & 17 & 85 & 79 \\
\hline 21 & 14 & 9 & 82 & 4 & 5 & 90 & 6 & 80 & 79 \\
\hline 22 & 14 & 5 & 59 & 0 & 3 & 10 & 1 & 15 & 80 \\
\hline 23 & 10 & 7 & 68 & 0 & 5 & 50 & 1 & 15 & 70 \\
\hline 24 & 10 & 7 & 68 & 1 & 4 & 50 & 3 & 15 & 83 \\
\hline 25 & 10 & 6 & 64 & 0 & 4 & 40 & 16 & 20 & 70 \\
\hline 26 & 10 & 7 & 68 & 0 & 5 & 50 & 1 & 15 & 70 \\
\hline 37 & 4 & 7 & 44 & 2 & 3 & 50 & 13 & 20 & 38 \\
\hline
\end{tabular}

Fuente elaboración propia, 2018
La tabla 6 muestra los resultados del prestest aplicado a la modalidad presencial que se mantuvo fuera de la "experimentación".

Tabla 6. Resultados del postest, grupo 2. Inglés intermedio nivel 5. Modalidad presencial.

\begin{tabular}{|c|c|c|c|c|c|c|c|c|c|}
\hline No. & $\begin{array}{l}\text { Rea- } \\
\text { ding } \\
(15)\end{array}$ & $\begin{array}{l}\text { Use of } \\
\text { Eng } \\
(10)\end{array}$ & $\begin{array}{l}\text { T L/U } \\
\text { of } E\end{array}$ & $\begin{array}{l}\mathrm{Wr} \\
(5)\end{array}$ & $\begin{array}{l}\mathrm{Wr} \\
(5)\end{array}$ & $\begin{array}{l}\text { Total } \\
\text { W }\end{array}$ & $\begin{array}{l}\text { List } \\
\text { (6) }\end{array}$ & $\begin{array}{l}\text { Total } \\
\text { List }\end{array}$ & Final \\
\hline 1 & 13 & 7 & 80 & 2 & 5 & 70 & 14 & 70 & 75 \\
\hline 2 & 8 & 6 & 56 & 1 & 4 & 50 & 9 & 45 & 70 \\
\hline 3 & 12 & 7 & 76 & 2 & 4 & 60 & 13 & 65 & 80 \\
\hline 4 & 11 & 7 & 72 & 1 & 4 & 50 & 10 & 50 & 65 \\
\hline 5 & 10 & 4 & 56 & 2 & 4 & 60 & 13 & 65 & 73 \\
\hline 6 & 13 & 8 & 84 & 2 & 5 & 70 & 16 & 80 & 93 \\
\hline 7 & 10 & 5 & 60 & 0 & 3 & 30 & 6 & 30 & 67 \\
\hline 8 & 7 & 9 & 64 & 2 & 5 & 70 & 16 & 80 & 86 \\
\hline 9 & 14 & 8 & 88 & 4 & 4 & 80 & 16 & 80 & 82 \\
\hline 10 & 9 & 7 & 64 & 0 & 4 & 40 & 16 & 40 & 90 \\
\hline 11 & 7 & 7 & 56 & 1 & 4 & 50 & 12 & 60 & 90 \\
\hline 12 & 5 & 4 & 36 & 0 & 5 & 50 & 16 & 60 & 91 \\
\hline 13 & 7 & 6 & 52 & 2 & 4 & 60 & 15 & 50 & 80 \\
\hline 14 & 7 & 8 & 60 & 2 & 3 & 50 & 15 & 25 & 80 \\
\hline 15 & 13 & 10 & 92 & 2 & 4 & 60 & 17 & 85 & 91 \\
\hline 16 & 8 & 7 & 60 & 1 & 3 & 40 & 9 & 45 & 90 \\
\hline 17 & 13 & 9 & 88 & 3 & 4 & 70 & 18 & 90 & 82 \\
\hline 18 & 7 & 7 & 56 & 1 & 4 & 50 & 12 & 60 & 80 \\
\hline 19 & 7 & 5 & 48 & 1 & 4 & 50 & 10 & 50 & 90 \\
\hline 20 & 14 & 9 & 92 & 4 & 5 & 90 & 17 & 85 & 89 \\
\hline 21 & 14 & 9 & 92 & 4 & 5 & 90 & 6 & 80 & 89 \\
\hline 22 & 14 & 5 & 76 & 0 & 3 & 30 & 1 & 15 & 90 \\
\hline 23 & 10 & 7 & 68 & 0 & 5 & 50 & 1 & 15 & 80 \\
\hline 24 & 10 & 7 & 68 & 1 & 4 & 50 & 3 & 15 & 83 \\
\hline 25 & 10 & 6 & 64 & 0 & 4 & 40 & 16 & 20 & 90 \\
\hline 26 & 15 & 10 & 100 & 4 & 4 & 80 & 15 & 30 & 80 \\
\hline 37 & 4 & 7 & 44 & 2 & 3 & 50 & 13 & 20 & 38 \\
\hline
\end{tabular}

Fuente elaboración propia, 2018 
En la tabla 7 se muestran los resultados del examen a la modalidad virtual, después de haber sido intervenido por la estrategia del uso de mensajería de WhatsApp.

Tabla 7. Resultados postest, grupo 1. Inglés intermedio nivel 5. Modalidad virtual

\begin{tabular}{|c|c|c|c|c|c|c|c|c|c|}
\hline No. & $\begin{array}{l}\text { Rea- } \\
\text { ding } \\
(15)\end{array}$ & $\begin{array}{l}\text { Use } \\
\text { of } \\
\text { Eng } \\
(10)\end{array}$ & $\begin{array}{l}T L / U \\
\text { of } E\end{array}$ & $\begin{array}{l}\mathrm{Wr} \\
\text { (5) }\end{array}$ & $\begin{array}{l}\text { Wr } \\
\text { (5) }\end{array}$ & $\begin{array}{l}\text { Total } \\
\text { W }\end{array}$ & $\begin{array}{l}\text { List } \\
\text { (6) }\end{array}$ & $\begin{array}{l}\text { Total } \\
\text { List }\end{array}$ & Final \\
\hline 1 & 13 & 7 & 80 & 2 & 5 & 70 & 14 & 90 & 95 \\
\hline 2 & 8 & 6 & 56 & 1 & 4 & 80 & 9 & 80 & 92 \\
\hline 3 & 12 & 7 & 76 & 2 & 4 & 60 & 13 & 95 & 96 \\
\hline 4 & 11 & 7 & 72 & 1 & 4 & 90 & 10 & 90 & 97 \\
\hline 5 & 10 & 4 & 86 & 2 & 4 & 80 & 13 & 85 & 98 \\
\hline 6 & 13 & 8 & 84 & 2 & 5 & 80 & 16 & 80 & 91 \\
\hline 7 & 10 & 5 & 80 & 0 & 3 & 30 & 6 & 90 & 89 \\
\hline 8 & 7 & 9 & 84 & 2 & 5 & 70 & 16 & 80 & 98 \\
\hline 9 & 14 & 8 & 88 & 4 & 4 & 80 & 16 & 80 & 87 \\
\hline 10 & 9 & 7 & 64 & 0 & 4 & 40 & 8 & 90 & 73 \\
\hline 11 & 7 & 7 & 56 & 1 & 4 & 50 & 12 & 90 & 94 \\
\hline 12 & 5 & 4 & 36 & 0 & 5 & 80 & 12 & 90 & 73 \\
\hline 13 & 7 & 6 & 52 & 2 & 4 & 80 & 10 & 80 & 70 \\
\hline 14 & 7 & 8 & 60 & 2 & 3 & 90 & 5 & 90 & 70 \\
\hline 15 & 13 & 10 & 92 & 2 & 4 & 90 & 17 & 85 & 94 \\
\hline 16 & 8 & 7 & 60 & 1 & 3 & 40 & 9 & 90 & 73 \\
\hline 17 & 13 & 9 & 88 & 3 & 4 & 70 & 18 & 90 & 72 \\
\hline 18 & 7 & 7 & 56 & 1 & 4 & 90 & 12 & 80 & 85 \\
\hline 19 & 7 & 5 & 48 & 1 & 4 & 50 & 10 & 50 & 99 \\
\hline 20 & 14 & 9 & 92 & 4 & 5 & 90 & 17 & 85 & 89 \\
\hline 21 & 14 & 9 & 92 & 4 & 5 & 90 & 16 & 30 & 80 \\
\hline 22 & 14 & 5 & 76 & 0 & 3 & 90 & 18 & 85 & 95 \\
\hline 23 & 10 & 7 & 68 & 0 & 5 & 80 & 17 & 96 & 80 \\
\hline 24 & 10 & 7 & 68 & 1 & 4 & 90 & 16 & 85 & 94 \\
\hline 25 & 10 & 6 & 64 & 0 & 4 & 90 & 16 & 80 & 81 \\
\hline 26 & 15 & 10 & 100 & 4 & 4 & 80 & 16 & 90 & 90 \\
\hline 37 & 4 & 7 & 44 & 2 & 3 & 90 & 15 & 80 & 88 \\
\hline 38 & 14 & 5 & 76 & 0 & 3 & 80 & 14 & 90 & 85 \\
\hline 39 & 10 & 7 & 68 & 0 & 5 & 90 & 14 & 80 & 80 \\
\hline 40 & 10 & 7 & 68 & 1 & 4 & 80 & 13 & 95 & 94 \\
\hline 41 & 10 & 6 & 64 & 0 & 4 & 90 & 14 & 80 & 91 \\
\hline 42 & 7 & 7 & 56 & 1 & 4 & 80 & 12 & 90 & 95 \\
\hline 43 & 7 & 5 & 48 & 1 & 4 & 80 & 15 & 50 & 99 \\
\hline 44 & 14 & 9 & 92 & 4 & 5 & 90 & 18 & 85 & 89 \\
\hline 45 & 14 & 9 & 82 & 4 & 5 & 76 & 18 & 80 & 80 \\
\hline
\end{tabular}

